\title{
Artigo/Article
}

\section{Análise espacial e determinação de áreas prioritárias para o controle da malária, no Estado no Tocantins, 2003-2008}

\author{
Spatial analysis and determination of priority areas for malaria control in the State of Tocantins, \\ from 2003 to 2008
}

\author{
Éldi Vendrame Parise ${ }^{1}$, Gessi Carvalho de Araújo ${ }^{2}$ e Renato Torres Pinheiro ${ }^{3}$
}

\begin{abstract}
RESUMO
Introdução: No Tocantins, a malária apresenta comportamento diferenciado entre as microrregiões, com predominância dos casos importados. Este estudo descreve a análise espacial da malária no estado, no período de 2003 a 2008, buscando identificar nas microrregiões a incidência de casos autóctones e importados, bem como a procedência destes últimos. Métodos: Trata-se de um estudo retrospectivo, pautado em dados secundários, que teve como fonte de dados o Sistema de Informações de Vigilância Epidemiológica - Malária (SIVEP-Malária), analisados através dos softwares estatísticos Epi Info versão 3.5.1. e Bioestat versão 5.0. Resultados: Constatou-se que a malária não teve distribuição homogênea em todos os municípios. A área de maior prioridade agregou municípios localizados nas microrregiões oeste do estado, fronteira com o Pará, onde também se concentram o maior número de casos autóctones. A associação entre os casos autóctones e importados e as espécies de Plasmodium mostrou uma diferença estatisticamente significativa $(\mathrm{G}=54,25 ; \mathrm{p}<0,0001)$. Das oito microrregiões, Miracema do Tocantins, Araguaína e Bico do Papagaio agruparam 75,8\% dos casos, e nessas, onze municípios se sobressaíram. Quanto à procedência, o Estado do Pará apresentou ampla distribuição com 85,5\% do total, seguido por Guiana Francesa com 7,4\%. Conclusões: Os resultados demonstraram a predominância dos casos importados e a diferença entre os municípios e microrregiões, apontando pela influencia de estados vizinhos na determinação das áreas de maior risco. Esses dados são importantes, pois contribuem para orientação e direcionamento das políticas públicas para o agravo no Tocantins.
\end{abstract}

Palavras-chaves: Análise espacial. Malária. Tocantins. Áreas de risco.

\begin{abstract}
Introduction: In Tocantins, the behavior of malaria differs between microregions, with predominance of imported cases. This study describes a spatial analysis on malaria in the state covering 2003 to 2008, which sought to identify the incidence of autochthonous and imported cases, and the origin of the latter, in the microregions. Methods: This was a retrospective study using secondary data. The data source was the epidemiological surveillance information system for malaria (SIVEP-Malária), and the data were analyzed using the Epi Info version 3.5.1 and Bioestat version 5.0 statistical software. Results: It was found that malaria was not homogeneously distributed in all municipalities. The area of highest priority comprised municipalities located in microregions in the west of the state, at the border of Pará, which also had the highest number of autochthonous cases. The association between the autochthonous and imported cases and the Plasmodium species showed a statistically significant difference $(G=54.25 ; \mathrm{p}<0.0001)$. Among the eight microregions, Miracema do Tocantins, Araguaína and Bico do Papagaio accounted for $75.8 \%$ of the cases and, among these, eleven municipalities stood out. Regarding provenance, the State of Pará showed widespread distribution with $85.5 \%$ of the total, followed by French Guiana with $7.4 \%$. Conclusions: These results demonstrated the predominance of imported cases and the difference between municipalities and microregions, and showed the influence of neighboring states in determining the areas of greatest risk. These data are important, since they contribute towards guiding and directing public policies regarding this disease in Tocantins.
\end{abstract}

Keywords: Spatial analysis. Malaria. Tocantins. Risk areas.

1. Diretoria de Vigilância em Saúde, Secretaria Municipal de Saúde, Palmas, TO. 2. Coordenação do Mestrado Profissional em Ciências da Saúde, Universidade Federal do Tocantins, Palmas, TO. 3. Coordenação de Medicina, Universidade Federal do Tocantins, Palmas, TO.

Endereço para correspondência: MsC. Éldi Vendrame Parise. Quadra 106 Sul, Alameda 14, Lote 06, 77020-062 Palmas,TO.

Tel: 5563 3026-2521; 5563 3218-5106

e-mail: eldiparise@gmail.com

Recebido para publicação em 21/08/2010

Aceito em 13/10/2010

\section{INTRODUÇÃO}

A malária é uma doença com perfil do mundo subdesenvolvido. Hoje em dia, ela é endêmica em 106 países e afeta um número de pessoas jamais conhecido na história ${ }^{1}$. Aproximadamente, 2,4 bilhões de pessoas vivem em áreas onde há risco de contrair a infecção ${ }^{2}$, especialmente na África, Ásia e América do Sul e Central. O maior foco de transmissão é a África Subsahariana, onde ocorrem $90 \%$ de todos os casos e mortes por malária no mundo ${ }^{1}$. Ocorrem cerca de 1,5 a 2,7 milhões de mortes a cada ano, a maioria causada por Plasmodium falciparum, sendo mais de um milhão em crianças menores de 5 anos de idade. $\mathrm{O}$ restante das mortes são, principalmente, em mulheres na sua primeira ou segunda gestação, adolescentes, adultos jovens, viajantes ${ }^{3}$, idosos e pessoas não-imunes, recémchegadas a áreas endêmicas ${ }^{4}$.

Os agentes etiológicos da malária humana são: Plasmodium falciparum, responsável pela terçã maligna; Plasmodium vivax, responsável pela terçã benigna; Plamodium malariae, responsável pela febre quartã e Plasmodium ovale, causador de uma forma de terçã benigna existente, principalmente, no continente africano. No Brasil, as duas primeiras espécies são mais prevalentes ${ }^{5}$, enquanto o Plasmodium malariae ocorre raramente ${ }^{6}$. O parasita apresenta dois ciclos de reprodução, sendo que a fase assexuada ocorre no hospedeiro intermediário (homem) e a fase sexuada no hospedeiro definitivo (mosquito Anopheles) ${ }^{7}$.

Geralmente, a enfermidade é transmitida pela picada de mosquitos, do gênero Anopheles, infectados e sua transmissão não se dá na mesma forma e rapidez em todas as áreas endêmicas. Sua distribuição está fundamentalmente baseada no hábito dos vetores, hábito das pessoas e modo de ocupação ${ }^{8,9}$.

De uma forma geral, as causas da malária são sempre múltiplas, um processo altamente complexo que envolve o agente, o hospedeiro e o ambiente, claramente associados à água, umidade, florestas e temperatura ${ }^{10}$. Normalmente, estão relacionadas à alta densidade anofélica, amplitude da malha hídrica, cobertura vegetal, desmatamentos, construção 
de hidroelétricas, estradas, sistema de irrigação, extração mineral e a presença de numerosos grupos populacionais, que somados às precárias condições de moradia, favorecem a aproximação do homem com o vetor e, consequentemente, uma rápida expansão da doenç $\mathrm{a}^{11}$. O processo migratório de populações procedentes de regiões do país onde não existe transmissão da malária para uma região altamente favorável à transmissão, é considerado um fator decisivo para o crescimento progressivo do número de casos e dispersão da doença9 .

Devido à ampla incidência e aos efeitos debilitantes, a malária é uma das doenças que mais contribuem para a decadência do homem da região amazônica, pois, além de reduzir os esforços físicos das pessoas na busca de seus recursos econômicos, diminui a capacidade produtiva e dificulta as condições de vida, interferindo no desenvolvimento socioeconômico da região ${ }^{12}$.

Sendo a malária um agravo que não se distribui de forma homogênea em todas as áreas ${ }^{9}$, e que possui características de uma doença focal, com áreas de transmissão natural, restrita a algumas regiões dentro de um mesmo país ${ }^{8}$, é de fundamental importância identificar as áreas de maior risco de transmissão para direcionar os esforços de forma mais precisa. Diante do exposto, este estudo se propõe a fazer uma análise espacial dos casos de malária no Tocantins, com o intuito de apontar as microrregiões e municípios que merecem maior atenção e com isso, contribuir com os serviços de saúde no planejamento das ações governamentais no combate à transmissão da doença e destinação adequada dos recursos necessários.

\section{MÉTODOS}

O presente trabalho refere-se ao levantamento dos casos de malária notificados nos 139 municípios do Tocantins, no período de 2003 a 2008. Trata-se de um estudo ecológico, descritivo, pautado em dados secundários, cuja menor unidade espacial adotada para análise foi o município, porém a área de estudo compreendeu todo o estado. Os dados notificados de malária foram disponibilizados pelo Sistema de Informações de Vigilância Epidemiológica - Malária (SIVEPMalária) ${ }^{6}$, da Secretaria de Vigilância em Saúde do Ministério da Saúde. Os dados censitários e as estimativas populacionais foram obtidos no site do Banco de Dados do Sistema Único de Saúde (DATASUS $)^{13}$, tendo como fonte o Instituto Brasileiro de Geografia e Estatística (IBGE), os quais foram utilizados como denominador no cálculo da incidência parasitária anual (IPA).

A descrição dos dados ficou concentrada naqueles que fizeram coleta de sangue para exame de plasmódio, através de busca ativa e busca passiva, já que estes representam apenas casos novos, e foram excluídos da discussão os exames laboratoriais classificados como lâminas de verificação de cura (LVC), pois estariam representando lâminas de uma mesma pessoa.

As variáveis que fundamentaram a discussão dos nossos estudos foram: a origem dos casos (autóctones e importados), o gênero, a incidência parasitária anual, a média da IPA nos seis anos por município e microrregião e a procedência dos casos importados.

A incidência parasitária anual, que representa o risco de transmissão da malária, foi expressa pelo número de exames positivos de cada município e/ou microrregião, dividido pela população da mesma área e período, multiplicado por mil habitantes, conforme proposto por Atanaka-Santos cols ${ }^{14}$. A média da IPA, no período 2003-2008, foi baseada na soma das IPAs de cada ano, dividido por seis (número de anos em estudo), semelhante aos procedimentos utilizados por Atanaka-Santos cols ${ }^{15}$ e Chaves e Rodrigues ${ }^{16}$.
A partir do resultado da IPA, os municípios e microrregiões foram classificados como alto risco (IPA maior que 49,9 casos por mil habitantes), médio risco (IPA entre 10 e 49,9 casos por mil habitantes), baixo risco (IPA entre 0,1 e 9,9 casos por mil habitantes) e sem risco (IPA igual a zero) $)^{5}$.

Considerando que a malária não se distribui de forma homogênea, junto à população, e que a tendência dos dados globais, muitas vezes não é representativa de todo o conjunto do estado, surge a necessidade de se fazer uma abordagem mais estratificada, por microrregião e por município, de modo a contemplar o cenário mais localizado possível. Primeiramente, os dados foram agrupados em oito microrregiões geográficas: Bico do Papagaio, Araguaína, Miracema do Tocantins, Porto Nacional, Rio Formoso, Gurupi, Dianópolis e Jalapão, conforme divisão estabelecida pelo IBGE $^{17}$ e, posteriormente, foram destacados aqueles municípios que representaram maior risco de transmissão.

Para reconhecer as microrregiões e município de maior relevância, foi somada a frequência de todos os anos em estudo, separados por classificação epidemiológica (autóctone e importada) e associadas à incidência parasitária anual. Os dados foram organizados em tabelas através do programa Microsoft Excel 2003, a fim de detectar os fenômenos de maior relevância e, os municípios que mereceram maior prioridade nas discussões foram identificados em mapa, permitindo com isso a visualização das áreas de maior risco no estado. Para verificar a associação entre as microrregiões e a classificação (autóctones e importados), nos indivíduos do sexo masculino, e também para avaliar as associações entre os casos autóctones e importados com a espécie de Plasmodium, foi utilizado o teste de G. A diferença entre as microrregiões, considerando as IPAs como variável dependente, foi analisada mediante o teste de Kruskal Wallis com teste a posteriori de Dunn. Para testar a independência entre as microrregiões e a frequência de Plasmodium falciparum e Plasmodium vivax, assim como entre as microrregiões e os casos autóctones e importados, foi utilizado o teste de qui-quadrado $\left(\chi^{2}\right)$. Todos os testes foram realizados considerando um nível de significância de $5 \%$. As análises estatísticas foram feitas com o auxílio do Epi Info versão 3.5.1 e Bioestat versão 5.0.

\section{Considerações éticas}

Seguindo as normas éticas para pesquisa envolvendo seres humanos, constantes na resolução ${ }^{\circ}$. 196/96 do Conselho Nacional da Saúde, o projeto de pesquisa foi submetido ao Comitê de Ética da Universidade Federal do Tocantins e aprovado em plenário no dia 10 de junho de 2008, sob o número 024/2008.

\section{RESULTADOS}

$\mathrm{Na}$ avaliação geral, constatamos que foram registrados 3.610 casos de malária. Desses, 1.237 (34,3\%) caracterizaram casos autóctones e $2.373(65,7 \%)$ casos importados, apresentando uma diferença estatisticamente significativa $\left(\chi^{2}=356,8 ; \mathrm{p}=0,0001\right)$. Quanto às espécies de Plasmodium, 2.647 (73,3\%) foram infecções por Plasmodium vivax, 787 (21,8\%) por Plasmodium falciparum, 173 (4,8\%) com infecções mistas, por P. falciparum e P. vivax e, apenas $3(0,1 \%)$ por Plasmodium malariae. A Tabela 1 mostra que houve uma associação estatisticamente significativa entre a classificação (autóctone e importada) e a espécie de Plasmodium ( $\mathrm{G}=54,25$; $\mathrm{p}<0,0001)$.

Quando analisado os casos autóctones, verificou-se que a proporção de infecções por Plasmodium vivax $(79,4 \%)$ é ainda maior que no total de casos; as infecções por Plasmodium falciparum representaram $18,8 \%$ e as infecções mistas, por P.falciparum e P. vivax, $1,8 \%$ da amostra. No que se refere aos casos de origem importada, 
TABELA 1 - Classificação epidemiológica dos casos de malária registrados no Estado do Tocantins, no período de 2003 a 2008, segundo as espécies do Plasmodium.

\begin{tabular}{|c|c|c|c|c|c|c|c|c|c|c|}
\hline \multirow[b]{4}{*}{ Classificação dos casos } & \multicolumn{10}{|c|}{ Espécie de Plasmodium } \\
\hline & \multicolumn{10}{|c|}{ falciparum + vivax } \\
\hline & \multicolumn{2}{|c|}{ vivax } & \multicolumn{2}{|c|}{ falciparum } & \multicolumn{2}{|c|}{ (mista) } & \multicolumn{2}{|c|}{ malarie } & \multicolumn{2}{|c|}{ total } \\
\hline & $\mathrm{n}^{\mathrm{o}}$ & $\%$ & $\mathrm{n}^{\mathrm{o}}$ & $\%$ & $\mathrm{n}^{\mathrm{o}}$ & $\%$ & $\mathrm{n}^{\mathrm{o}}$ & $\%$ & $\mathrm{n}^{\mathrm{o}}$ & $\%$ \\
\hline Autóctones & 982 & 79,4 & 233 & 18,8 & 22 & 1,8 & - & - & 1.237 & 34,3 \\
\hline Importados & 1.665 & 70,2 & 554 & 23,4 & 151 & 6,4 & 3 & 0,1 & 2.373 & 65,7 \\
\hline Total & 2.647 & 73,3 & 787 & 21,8 & 173 & 4,8 & 3 & 0,1 & 3.610 & 100,0 \\
\hline
\end{tabular}

o Plasmodium vivax agrupou 70,2\%, Plasmodium falciparum $23,4 \%$, as infecções mistas, por P. falciparum e P. vivax 6,4\% e o Plasmodium malariae $0,1 \%$. Importante destacar que $62,9 \%$ dos casos infectados por Plasmodium vivax, 70,4\% infectados por Plasmodium falciparum e $87,3 \%$ dos casos infectados por malária mista, se referem aos casos de origem importada.

Ao separar os casos de malária por espécie de Plasmodium e microrregião, constatou-se que o Plasmodium falciparum e Plasmodium vivax foram encontrados nas oito microrregiões do estado, e houve uma associação significativa entre elas $\left(\chi^{2}=90,28\right.$; $\mathrm{p}<0,0001)$; contudo, as infecções mistas apareceram somente em seis microrregiões, exceto Dianópolis e Jalapão, e as infecções por Plasmodium malariae, apenas em Miracema do Tocantins.

Na Tabela 2, estão representadas as amostras de sangue examinadas, para o controle da malária, o número e percentual de positividade, a média da incidência parasitária anual e a classificação dos casos por autóctones e importados, de acordo com as oito microrregiões geográficas.

A microrregião de Miracema do Tocantins apresentou o maior registro com 29,5\% do total de casos, seguido pela microrregião de Araguaína, Bico do Papagaio e Porto Nacional com 25,8\%, 20,4\% e $11,5 \%$, respectivamente, os quais representaram $87,3 \%$ do total de casos. As demais microrregiões, Rio Formoso, Gurupi, Dianópolis e Jalapão, registraram apenas $12,7 \%$ do total de casos, sendo Jalapão, a microrregião com o menor percentual, $0,4 \%$ do total.

Com relação aos casos autóctones, $86,4 \%$ dos casos ficaram agrupados em três microrregiões. Miracema do Tocantins sobressaiuse com 59,7\% da autoctonia, seguida por Bico do Papagaio e Araguaína com $14,2 \%$ e 12,5\%, respectivamente $\left(\chi^{2}=2689 ; \mathrm{p}<0,0001\right)$. Quando analisados os casos importados, a microrregião de Araguaína, que estava em terceiro lugar na autoctonia, passou a ocupar o primeiro lugar nos importados, com 32,8\% do total de casos, seguida pelo Bico do Papagaio com 23,7\%, Porto Nacional com 14,7\% e Miracema do Tocantins com $13,8 \%$. Esta que estava em primeiro lugar, em relação aos casos autóctones, passou a ocupar o quarto lugar no registro dos casos importados. Verificamos uma diferença significativa nos casos importados por microrregião $\left(\chi^{2}=1815 ; \mathrm{p}<0,0001\right)$.

Quanto ao risco que a malária oferece, em cada microrregião, a média da IPA variou entre $0,03 / 1.000$ a $1,25 / 1.000$ habitantes. A microrregião de Miracema do Tocantins que apresentou o maior IPA em 2003 (3,47/1.000 habitantes) registrou apenas 0,07/1.000 habitantes em 2008, com média de 1,25/1.000; o Bico do Papagaio que tinha 1,28/1.000 habitantes em 2003 reduziu para $0,46 / 1.000$ habitantes em 2008, e média de 0,65/1.000; e Araguaína que registrou $1,17 / 1.000$ habitantes em 2003, passou para 0,17/1.000 habitantes em 2008, com média de 0,62/1.000. Ao calcular o índice de variação dos casos, constatou-se que a microrregião com maior redução durante o período em estudo foi Miracema do Tocantins (-97,9\%), seguido de Rio Formoso (-92,4\%), Porto Nacional (-86,9\%), Araguaína (-85,6\%) e Bico do Papagaio (-63,6\%). Quando analisadas as IPAs, nas diversas microrregiões, houve variação estatisticamente significativa $(H=20,73 ; \mathrm{p}=0,0004)$. $\mathrm{O}$ teste a posteriori de Dunn, utilizado para comparar os pontos médios mostrou uma diferença significativa (diferença $=37,83 ; \mathrm{p}<0,05$ ), unicamente entre as microrregiões de Miracema e Porto Nacional.

Quando foram separados os casos de malária por gênero, constatouse que em todas as microrregiões geográficas, os indivíduos masculinos sempre representaram a maior proporção, totalizando $77 \%$ dos casos. Ao analisá-los, separadamente, por classificação epidemiológica, a média dos indivíduos masculinos ficou em $69,9 \%$ nos casos autóctones

TABELA 2 - Número de lâminas examinadas para malária registradas no Estado do Tocantins no período de 2003 a 2008, número e percentual de positividade, média da incidência parasitária anual e classificação dos casos por autóctones e importados, segundo microrregião geográfica.

\begin{tabular}{|c|c|c|c|c|c|c|c|c|}
\hline \multirow[b]{3}{*}{ Microrregiões } & \multicolumn{3}{|c|}{ Amostras de sangue } & \multirow{3}{*}{$\begin{array}{c}\text { Média } \\
\text { IPA/1.000 }\end{array}$} & \multirow{2}{*}{\multicolumn{2}{|c|}{ Autóctone }} & \multirow{2}{*}{\multicolumn{2}{|c|}{ Importados }} \\
\hline & \multirow{2}{*}{$\begin{array}{c}\text { examinadas } \\
\mathrm{n}^{\circ}\end{array}$} & \multicolumn{2}{|c|}{ positivas } & & & & & \\
\hline & & $\mathrm{n}^{\mathrm{o}}$ & $\%$ & & $\mathrm{n}^{\mathrm{o}}$ & $\%$ & $\mathrm{n}^{\mathrm{o}}$ & $\%$ \\
\hline Bico do Papagaio & 4.400 & 738 & 20,4 & 0,65 & 176 & 14,2 & 562 & 23,7 \\
\hline Araguaína & 5.140 & 933 & 25,8 & 0,62 & 155 & 12,5 & 778 & 32,8 \\
\hline Miracema do Tocantins & 4.229 & 1.065 & 29,5 & 1,25 & 738 & 59,7 & 327 & 13,8 \\
\hline Porto Nacional & 2.727 & 415 & 11,5 & 0,25 & 67 & 5,4 & 348 & 14,7 \\
\hline Rio Formoso & 1.703 & 339 & 9,4 & 0,52 & 38 & 3,1 & 301 & 12,7 \\
\hline Gurupi & 543 & 82 & 2,3 & 0,11 & 30 & 2,4 & 52 & 2,2 \\
\hline Dianópolis & 221 & 24 & 0,7 & 0,03 & 22 & 1,8 & 02 & 0,1 \\
\hline Jalapão & 41 & 14 & 0,4 & 0,03 & 11 & 0,9 & 03 & 0,1 \\
\hline Total & 19.004 & 3.610 & - & - & 1.237 & - & 2.373 & - \\
\hline
\end{tabular}

Distribuição autóctone: $\left(\chi^{2}=2689 ; \mathrm{p}<0,0001\right)$, distribuição importados: $\left(\chi^{2}=1815\right.$; $\left.\mathrm{p}<0,0001\right)$, média IPAs entre microrregiões: $(\mathrm{H}=20,73 ; \mathrm{p}=0,0004)$ IPA: incidência parasitária anual. 
e $80,6 \%$ nos casos importados. A associação entre as microrregiões e os casos autóctones e importados, nos indivíduos do sexo masculino, foi estatisticamente significativa ( $\mathrm{G}=637,05 ; \mathrm{p}<0,0001)$.

No período de 2003 a 2008, o Tocantins registrou malária em 96 (69,1\%) municípios. Desses, 83 registraram casos autóctones e 57 registraram casos importados, sendo que na maioria ocorreram as duas formas. No primeiro ano em estudo, foram $55(39,6 \%)$ municípios com registro de casos, e em 2008 , apenas $26(18,7 \%)$; uma redução de $52,7 \%$ dos municípios com circulação do parasita. E o mais importante é que, desses que registraram malária em 2008 somente nove $(6,5 \%)$ registraram casos autóctones.

Na Figura 1 está representada a divisão do Estado do Tocantins em microrregiões geográficas e a localização dos onze municípios que se destacaram em função da quantidade de malária. Observou-se que oito dos municípios que registraram a maior frequência de casos estão situados às margens do rio Araguaia, lado oeste do estado, e fazem divisa com o Estado do Pará.

Os municípios que merecem ser evidenciados na descrição, pela maior frequência de casos e maior média geral da IPA, são os que fazem parte da microrregião de Miracema do Tocantins, seguidos pelos do Bico do Papagaio e Araguaína (Tabela 3).

Na microrregião de Miracema do Tocantins, os municípios com maior número de casos de malária foram: Caseara (280 autóctones e 161 importados), Araguacema (214 autóctones e 26 importados) e Marianópolis do Tocantins (181 autóctones e 22 importados). Esses municípios registraram a maior média e desvio padrão da IPA, entre os onze municípios com maior frequência de casos, com 18,01/1.000 habitantes; 6,96/1.000 habitantes; 9,05/1.000 habitantes, respectivamente. No início da série, os três municípios estavam enquadrados na classificação de médio risco ( 10 a 49,9 casos/ 1.000 habitantes). Já em 2008, Caseara e Araguacema ficaram classificados
Esperantina

Araguatins

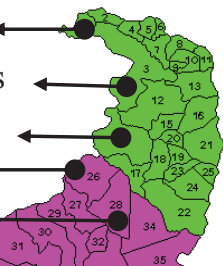

MICRORREGIÕES

- Bico do Papagaio

- Araguaína

- Miracema do Tocantins

Porto Nacional

- Rio Formoso

- Gurupi

- Dianópolis

- Jalapão

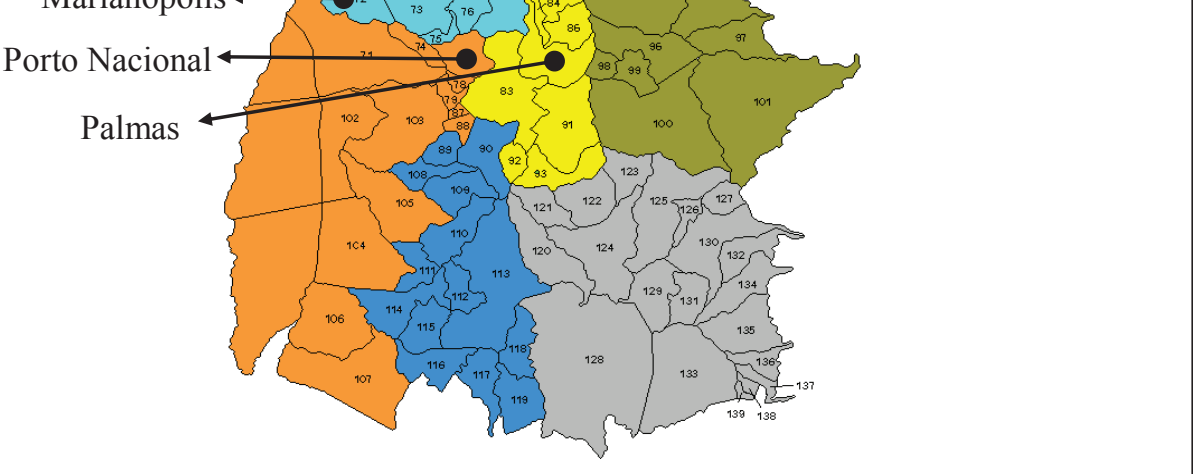

FIGURA 1 - Divisão geográfica do Estado do Tocantins, por microrregião e localização dos onze municípios

como de baixo risco (IPA entre 0,1 e 9,9 casos por mil habitantes) e Marianópolis do Tocantins, sem risco $($ IPA $=0)$. Neste último ano, dos 17 municípios que fazem parte desta microrregião, apenas Caseara e Araguacema permaneceram com transmissão autóctone ( 3 e 1 casos, respectivamente), os quais também registraram o mesmo número de casos importados.

Na microrregião do Bico do Papagaio, os municípios com maior frequência, porém, bem menor que a microrregião anterior, foram: Araguatins (70 casos autóctones e 268 importados), Ananás (15 autóctones e 58 importados) e Esperantina (11 autóctones e 50 importados). A média da IPA ficou em 2,02/1.000 habitantes; 1,10/1.000 habitantes; $1,16 / 1.000$ habitantes, respectivamente. Desde o início da série, os três municípios ficaram enquadrados na classificação de baixo risco. Em 2008, dos 25 municípios que fazem parte desta

TABELA 3 - Incidência parasitária anual, média e desvio padrão da IPA dos municípios com maior registro de casos de malária, no período 2003 a 2008 , segundo microrregião geográfica.

\begin{tabular}{llrrrrrrr}
\hline & & \multicolumn{4}{c}{ Incidência parasitária anual } & \multicolumn{2}{c}{ Média \pm DP } \\
\cline { 2 - 6 } Microrregião & Municípios & 2003 & 2004 & 2005 & 2006 & 2007 & 2008 & IPA/1.000 \\
\hline Bico do Papagaio & Ananás & 2,05 & 0,78 & 1,51 & 0,58 & 0,53 & 1,14 & $1,10 \pm 0,6$ \\
& Araguatins & 4,38 & 1,48 & 2,35 & 0,93 & 1,62 & 1,35 & $2,02 \pm 1,2$ \\
& Esperantina & 2,37 & 1,70 & 1,08 & 0,83 & 0,61 & 0,36 & $1,16 \pm 0,8$ \\
\hline Araguaína & Araguaína & 1,76 & 1,32 & 0,67 & 0,53 & 0,42 & 0,28 & $0,83 \pm 0,6$ \\
& Piraquê & - & 7,12 & 3,62 & 3,49 & 0,33 & - & $2,43 \pm 2,8$ \\
& Xambioá & 3,63 & 0,89 & 2,38 & 4,30 & 1,84 & 0,45 & $2,25 \pm 1,5$ \\
\hline Miracema do TO & Araguacema & 24,38 & 12,69 & 2,33 & 0,16 & 1,84 & 0,36 & $6,96 \pm 9,7$ \\
& Caseara & 46,53 & 29,35 & 22,31 & 3,94 & 4,71 & 1,25 & $18,01 \pm 18,0$ \\
& Marianópolis do TO & 32,42 & 11,12 & 7,83 & 2,46 & 0,45 & - & $9,05 \pm 12,8$ \\
\hline Porto Nacional & Palmas & 0,48 & 0,38 & 0,41 & 0,14 & 0,15 & 0,07 & $0,27 \pm 0,2$ \\
\hline Rio Formoso & Paraíso do TO & 1,42 & 1,48 & 2,13 & 0,54 & 0,57 & 0,14 & $1,05 \pm 0,7$ \\
\hline
\end{tabular}

IPA: Incidência parasitária anual, DP: média e desvio padrão das IPAs, TO: Tocantins. 
microrregião, cinco municípios permaneceram com transmissão autóctone, com um caso de cada, e apenas um registrou três casos. Ao mesmo tempo, verificou-se que estes municípios também registraram casos importados, o que favoreceu o surgimento da autoctonia.

Na microrregião de Araguaína, os municípios com maior número de casos foram: Araguaína (25 autóctones e 586 importados), Xambioá (22 autóctones e 143 importados) e Piraquê (51 autóctones). Nos municípios de Araguaína e Xambioá, 95,9\% e 86,7\%, respectivamente, são de procedência importada, enquanto em Piraquê, todos os casos registrados são autóctones; representando risco para este último. Entretanto, a IPA dos seis anos em estudo e também a média da IPA, demonstrou que os três municípios sempre ficaram enquadrados na classificação de baixo risco. No último ano da série, nenhum município da microrregião registrou transmissão autóctone e, apenas quatro municípios registraram casos importados.

Nas microrregiões de Porto Nacional e Rio Formoso, merecem discussão a capital do estado, Palmas e o município de Paraíso do Tocantins, uma vez que respondem por $15,6 \%$ do total de casos. Os dois municípios registraram malária em todos os anos da série, porém, $97,4 \%$ e 99,6\%, respectivamente, são de procedência importada. Palmas registrou ao todo 8 casos autóctones (sendo o último em 2005) e 301 importados; Paraíso do Tocantins registrou 1 caso autóctone e 253 importados. No entanto, em 2008, foram registrados apenas 13 casos importados para Palmas e 6 casos para Paraíso do Tocantins, indicativo de que a malária está sob controle.

Na Tabela 4, encontram-se distribuídos os casos de malária importada, segundo estados brasileiros ou país de procedência. Do total de casos importados, 2.143 (90,3\%) foram infectados no Brasil e $230(9,7 \%)$ em outros países. A maioria dos casos, 2.135 (89,9\%) originou-se dos estados que compõem a Amazônia brasileira, $4(0,2 \%)$ casos nos demais estados do nordeste, $3(0,1 \%)$ casos nos estados do sudeste, $1(0,04 \%)$ caso no centro-oeste e o restante, $230(9,7 \%)$ casos fora do país.

TABELA 4 - Distribuição anual dos casos de malária importada registrada no Estado do Tocantins, no período de 2003 a 2008, segundo estado ou país de procedência.

\begin{tabular}{|c|c|c|c|c|c|c|c|c|}
\hline \multirow[b]{2}{*}{ Estado/país de procedência } & \multicolumn{6}{|c|}{ Ano } & \multicolumn{2}{|c|}{ Total de casos } \\
\hline & 2003 & 2004 & 2005 & 2006 & 2007 & 2008 & $\mathrm{n}^{\mathrm{o}}$ & $\%$ \\
\hline Amazonas & 1 & 4 & 4 & 2 & 2 & 2 & 15 & 0,6 \\
\hline Amapá & 4 & 5 & 6 & 3 & - & 1 & 19 & 0,8 \\
\hline Bahia & - & 1 & - & - & 1 & - & 2 & 0,1 \\
\hline Espírito Santo & - & - & - & - & - & 1 & 1 & 0,04 \\
\hline Goiás & 1 & - & - & - & - & - & 1 & 0,04 \\
\hline Maranhão & 6 & 7 & 6 & 1 & 2 & 4 & 26 & 1,1 \\
\hline Minas Gerais & 2 & - & - & - & - & - & 2 & 0,1 \\
\hline Mato Grosso & 6 & 4 & 1 & 1 & 1 & 1 & 14 & 0,6 \\
\hline Pará & 629 & 455 & 419 & 227 & 192 & 108 & 2.030 & 85,5 \\
\hline Paraíba & 1 & - & 1 & - & - & - & 2 & 0,1 \\
\hline Rondônia & 3 & 9 & 3 & 4 & 6 & 2 & 27 & 1,1 \\
\hline Roraima & - & 1 & 1 & - & - & 2 & 4 & 0,2 \\
\hline África do Sul & - & - & - & 1 & 1 & - & 2 & 0,1 \\
\hline Angola & - & 1 & - & 1 & - & 2 & 4 & 0,2 \\
\hline EUA & - & - & - & - & - & 1 & 1 & 0,04 \\
\hline França & - & - & - & - & - & 2 & 2 & 0,1 \\
\hline Guiana & 5 & 1 & - & - & 2 & 1 & 9 & 0,4 \\
\hline Guiana Francesa & 27 & 29 & 29 & 42 & 30 & 19 & 176 & 7,4 \\
\hline Suriname & 5 & 4 & 8 & 3 & 5 & 6 & 31 & 1,3 \\
\hline Venezuela & 1 & - & 3 & - & 1 & - & 5 & 0,2 \\
\hline Total & 691 & 521 & 481 & 285 & 243 & 152 & 2.373 & - \\
\hline
\end{tabular}

Pode-se verificar a influência expressiva do Estado do Pará na distribuição da malária para o Tocantins, com $85,5 \%$ dos casos.

No período em estudo, o Tocantins registrou malária de origem importada em 57 municípios. O Estado do Pará esteve presente em 54 (94,7\%) deles, Guiana Francesa em 20 (35,1\%) municípios, Maranhão em 13 (22,8\%), Mato Grosso e Suriname em 9 (15,8\%) cada, Amazonas em 7 (12,3\%) municípios e os demais estados e países citados estiveram presente em menos de seis municípios de cada.

Os municípios tocantinenses com maior número de casos importados foram: Araguaína com 586 casos, Palmas com 301, Araguatins com 268, Paraíso do Tocantins com 253, Caseara com 161, Xambioá com 143, Ananás com 58 e Esperantina com 50 casos. Desses, 89,1\%, 75,4\%, 82,8\%, 96\%, 100\%, 97,2\%, 86,2\% e $72 \%$, respectivamente, tiveram origem no Estado do Pará. Outros municípios, também, registraram malária importada do Pará; porém, a frequência ficou abaixo de 50 casos de cada.

Com relação à malária importada de outros países, Guiana Francesa ficou em primeiro lugar, com $176(7,4 \%)$ casos e Suriname em segundo lugar, com 31 (1,3\%) casos. Os dois países responderam por $8,7 \%$ do total de casos registrados no Tocantins e por $90 \%$ dos casos importados de fora do Brasil; a maior parte dos casos é procedente de áreas de garimpo, agropecuária e florestas.

\section{DISCUSSÃo}

De uma forma geral, o estudo demonstrou que, no Estado do Tocantins, existe uma diferença significativa, entre o total de casos autóctones e importados (Tabela 1), reflexo das peculiaridades ambientais, ecológicas e socioeconômicas da região, e do fluxo constante de migrantes para áreas de maior endemicidade. Este resultado foi similar aos obtidos no município de Belém/Pará ${ }^{18}$ e no Estado de Santa Catarina ${ }^{19}$; no entanto, diferente do que ocorre atualmente nos demais estados que fazem parte da Amazônia Legal.

Com relação às espécies de Plasmodium, foi possível diagnosticar que a maioria dos indivíduos foi infectada por Plasmodium vivax, semelhante aos resultados encontrados em Mato Grosso ${ }^{14}$, Roraima ${ }^{16}$, Amazonas ${ }^{20}$, Rondônia ${ }^{21,22}$, Pará $^{23}$ e Guatemala ${ }^{24}$.

Ao comparar autóctones e importados, constatou-se que nestes, a proporção de infecções por Plasmodium falciparum e mista são maiores que o total geral, fortalecendo as informações de Barbieri ${ }^{7}$ e Monte-Mór ${ }^{25}$ quando relacionam este parasita à mobilidade espacial e à expansão do processo de ocupação da terra.

Na região Amazônica, os altos índices de Plasmodiumfalciparum referem-seaosgarimpos desorganizados, assentamentos recentes em projetos de colonização, comunidades colocadas em contato direto e duradouro com a mata nativa, onde os vetores da doença vivem e proliferam de forma natural ${ }^{26}$.

Esse padrão de comportamento, na busca por atividades de campo fora de sua 
localidade de residência, é muito mais frequente em indivíduos do sexo masculino, tanto que, em nossos estudos, os homens representaram a maioria das infecções em todas as microrregiões. Yeshiwondim cols ${ }^{27}$ também tiveram esta constatação, e atribuíram este fato às diferentes taxas de exposição, maior mobilidade, trabalhos relacionados com atividades nos campos e permanência em áreas de maior risco de transmissão.

Ao estratificar o estado em microrregiões, observou-se uma grande variação na dinâmica da malária (Tabela 2). Enquanto a microrregião de Jalapão registrou apenas quatorze casos durante os seis anos, a microrregião de Miracema do Tocantins registrou mais de mil casos; demonstrando que a malária não se distribuiu de forma homogênea junto à população, assim como tem sido descrito por Tauil ${ }^{9}$, na Amazônia Brasileira e por Dias ${ }^{8}$, no Estado de Roraima.

Ficou evidente que a malária é uma doença de ocorrência local e focal ${ }^{8}$, que acompanha o grau de migração, contato direto com as matas, águas e, consequentemente, com os vetores ${ }^{7}$. No Tocantins, a infecção se concentrou mais no lado oeste-centro-norte do estado (Figura 1), e os municípios com maior incidência encontram-se localizados às margens do rio Araguaia e/ou passaram por projetos de assentamentos de reforma agrária, corroborando com os dados de Atanaka-Santos cols ${ }^{15}$, Marques e Gutierrez ${ }^{28}$ e os descritos pelo Ministério da Saúde ${ }^{4}$.

A microrregião de maior incidência foi Miracema do Tocantins. A mesma registrou a maior frequência de casos, maior média da IPA (Tabela 2) e 94,3\% dos seus registros autóctones estão notificados em municípios que fazem parte da Área de Preservação Ambiental Ilha do Bananal/Cantão, oeste do estado, formados por planícies, ilhas, canais e matas alagadas de várzea, com uma vegetação de cerrado, floresta Amazônica, inundável e perene, compatível com a estratificação de médio e alto risco estabelecido por Marques e Gutierrez $^{28}$ e pela descrição feita por Forattini cols ${ }^{29}$.

Outro fator que também pode ter influenciado na transmissão é a grande quantidade de praias fluviais que se formam às margens dos rios, no período em que o nível da água se encontra baixo. A pesquisa indicou que $63,1 \%$ dos casos de malária autóctones ocorreram em municípios que possuem em sua área locais de praias fluviais; assemelhando-se aos dados de Alves $\operatorname{cols}^{30}$ ao descreverem que em Campinas 16,8\% dos casos estavam relacionados ao lazer; a Machado cols $^{19}$, quando descreveram que os casos autóctones em Santa Catarina se concentraram nas localidades com grande potencial turístico; aos dados do Ministério da Saúde ${ }^{12}$ e Barbieri ${ }^{31}$, ao referir que a entrada de grandes contingentes humanos provoca modificação do equilíbrio natural do meio, alterando as características reprodutivas, o habitat dos vetores e mesmo os seus hábitos alimentares, impulsionando-os a se alimentar mais de sangue humano do que de outros animais.

O Tocantins possui 64 municípios que possibilitam a formação de praias às margens dos rios, as quais favorecem o fluxo intenso e aglomeração de pessoas no período de junho a setembro. Esses ambientes, por apresentarem águas lentas e proximidade com a vegetação, podem estabelecer locais propícios para o desenvolvimento dos anofelinos e, consequentemente, provocar maior incidência da doença. Isso porque a presença de apenas uma pessoa portadora do parasito poderá estabelecer rapidamente a transmissão e provocar uma epidemia sem que se perceba de imediato sua extensão ${ }^{25}$.

Quanto aos casos importados, os municípios que mais registraram foram aqueles localizados próximos à fronteira do Pará, mais especificamente nas microrregiões de Araguaína e Bico do Papagaio (Figura 1), cujas características climáticas e ambientais possuem forte influência Amazônica. A maioria desses casos é de pessoas que possuem famílias, fixam residência nas cidades tocantinenses e saem em busca de oportunidades em outros estados ou países. Quando começam sentir os primeiros sintomas, retornam junto às famílias para se tratar, o que resulta no grande número de casos de origem importada para o estado, e como se constatou, a maioria do sexo masculino.

A influência que o Pará exerce sobre o Tocantins com relação à disseminação da malária, detectada em nossa pesquisa, já foi constatada por Marques cols ${ }^{32}$, em 1986, quando o Tocantins ainda fazia parte do Estado de Goiás. Naquele ano, o autor descreveu que $73,2 \%$ dos casos registrados em Goiás eram provenientes de municípios do Pará, principalmente de São Felix do Xingu, Santana do Araguaia, Conceição do Araguaia e Marabá. Parte considerável daqueles casos foi registrada em municípios hoje tocantinenses (Araguaína, Paraíso do Tocantins, Gurupi, Guaraí, Araguatins, Tocantinópolis e Porto Nacional).

Estudos publicados, recentemente, informam que o Pará ainda é considerado uma área endêmica para malária. $\mathrm{O}$ estado possui $26,6 \%$ dos municípios com potencial elevado de transmissão, sendo $21,7 \%$ com IPA igual ou superior a 50 (alto risco). A região apresenta características ambientais, ecológicas e socioeconômicas que favorecem a interação do plasmódio e dos anofelinos e, a migração intensa nos processos de colonização, o crescimento populacional desordenado ao longo das florestas, rios e rodovias, determinam diferentes níveis de transmissão endêmica ${ }^{23}$.

Geograficamente, o Tocantins faz fronteira com o Pará. O fluxo de pessoas entre esses dois estados acaba sendo frequente em função da demanda de mão de obra oferecida pelo Pará, nas áreas ligadas à agricultura, pecuária e exploração vegetal. Esse elevado número de casos importados oriundos do Pará, possivelmente, tenha ocorrido devido à migração humana para essas áreas em busca de trabalho e seu posterior regresso à sua cidade de origem, principalmente por colonizadores e trabalhadores rurais, assim como tem explicado Marques e $\mathrm{cols}^{32}$.

Os resultados encontrados com relação à Guiana Francesa e Suriname, de serem responsáveis por quase a totalidade dos casos importados de fora do Brasil, assemelham-se aos dados registrados na região extra-amazônica, descritos pelo Ministério da Saúde ${ }^{33}$. Quanto à procedência dos casos, referida às áreas de garimpo, agropecuária e florestas, já tem sido discutida por Barbieri ${ }^{7}$, quando descreve que os indivíduos que desenvolvem atividades em assentamento, desmatamento e exploração mineral estão mais expostos à doença, em função da abundância dos vetores contaminados, forma de ocupação do solo, que facilita a exposição extradomiciliar e a precariedade das habitações, as quais não oferecem proteção e acabam proporcionando maior interação entre o homem e os vetores transmissores.

Considerando a frequência e a procedência da malária, acreditase que a diminuição dos casos importados no Tocantins também tenha sofrido influência do Estado do Pará, uma vez que, conforme se verifica indicativo de melhora nos índices da malária nesse estado, o Tocantins recebe os mesmos reflexos e reduz os casos importados. Ao contrário, em 2005, quando o Pará registrou um incremento de $12,4 \%$ dos casos de malária, o Tocantins, embora apresentasse queda no número de casos, também demonstrou curva ascendente no índice de lâminas parasitadas ${ }^{34}$.

Oliveira-Filho e Martinelli ${ }^{23}$ afirmam que o número de municípios com risco de transmissão de malária no Pará está diminuindo. Segundo eles, a disponibilidade dos serviços de saúde oferecidos aos pacientes, em municípios com dificuldade de acesso, pode ter influenciado positivamente na redução dos casos e, consequentemente, ter reduzido o percentual de municípios em situação de risco. Este benefício pode ter se estendido ao Tocantins, uma vez que a maioria dos 
casos importados é procedente daquele estado e, como se constatou vem demonstrando importante redução.

Com relação à redução dos casos autóctones, assim como a ausência da transmissão em alguns municípios tocantinenses, poderá estar refletindo o avanço desses municípios na melhoria das ações de controle da malária, adotadas em ação conjunta e permanente com o governo e a sociedade, que resultou no fortalecimento dos níveis regionais elocais de atenção à saúde. Acreditamos que as ações que influenciaram na melhoria dos indicadores de malária nos municípios, tiveram como alicerce as estratégias do Programa Nacional de Controle da Malária (PNCM), agregadas às sugestões das secretarias estaduais e municipais de saúde $\mathrm{e}$ do Comitê Técnico de Acompanhamento, que propuseram e colocaram em prática o fortalecimento da vigilância da malária; apoio à estruturação dos sistemas locais de saúde; diagnóstico rápido e tratamento correto; capacitação de recursos humanos; educação em saúde; comunicação e mobilização social em parceria com outras instituições, a fim de obter a participação da população nas ações; controle seletivo de vetores; pesquisa; monitoramento do programa e sustentabilidade política ${ }^{35}$.

Sabendo-se que a maioria dos casos de malária registrados no Tocantins é de origem importada, de trabalhadores do campo que saem em busca de trabalho em outros estados e/ou países para o sustento de suas famílias, e que esse fluxo se mantém constante, servindo de portadores ativos do Plasmodium para o Tocantins, verifica-se a importância de se aprimorar estratégias de educação popular sobre medidas de prevenção e despertar a consciência nos pacientes pela busca do atendimento em unidades de saúde logo no início dos primeiros sintomas, a fim de evitar o surgimento de casos introduzidos, decorrente dos importados.

\section{CONFLITO DE INTERESSE}

Os autores declaram não haver nenhum tipo de conflito de interesse no desenvolvimento do estudo.

\section{SUPORTE FINANCEIRO}

Secretaria de Ciência e Tecnologia (SECT) do Estado do Tocantins.

\section{REFERÊNCIAS}

1. Finkel M. Bedlam in the blood malaria. Natl Geogr Mag 2007; 212:32-67.

2. Organização Mundial de Saúde. Tratamento da malária grave e complicada: guia de condutas práticas. 2a ed. Brasília: Organização Mundial de Saúde; 2000.

3. Jardínes IQ. Malaria: características generales y situación actual em Cuba y lãs Américas. Biblioteca Virtual de Vigilância em Salud. 6: 2001. [Acesso em 29 jan. 2009]. Disponível em http://www.bvs.sld.cu/uats/rtv_files/rtv0501.htm/.

4. Ministério da Saúde. Fundação Nacional de Saúde. Manual de Terapêutica da Malária. 6a ed. Brasília: Ministério da Saúde; 2001.

5. Ministério da Saúde. Secretaria de Vigilância em Saúde. Departamento de Vigilância Epidemiológica. Ações de controle da malária: manual para profissionais de saúde na atenção básica. $2^{\mathrm{a}}$ ed. Brasília: Ministério da Saúde; 2006.

6. Ministério da Saúde. Secretaria de Vigilância em Saúde. Sistema de Informação de Vigilância Epidemiológica. Dados de malária. [Acesso em 10 maio 2009]. Disponível em http://saúde.gov.br/sivep_malária/.

7. Barbieri AF. Uso do solo e prevalência de malária em uma região da Amazônia Brasileira. Cad Geografia 2005; 15:9-30.

8. Dias RC. Uma contribuição ao estudo da malária no Estado de Roraima e sua associação com a precipitação pluviométrica no período de 1985 a 1996. [Tese de Doutorado]. [Rio de Janeiro (RJ)]: Escola Nacional de Saúde Pública, Fundação Osvaldo Cruz; 2003.

9. Tauil PL. Avaliação de uma nova estratégia de controle da malária na Amazônia Brasileira. [Tese de Doutorado]. [Brasília (DF)]: Universidade de Brasília; 2002.
10. Tosta CE. Coadaptation and malaria control. Mem Inst Oswaldo Cruz 2007; 102:385-404.

11. Ministério da Saúde. Secretaria de Atenção à Saúde, Departamento de Atenção Básica. Brasil.Vigilância em Saúde: dengue, esquistossomose, hanseníase, malária, tracoma e tuberculose. 2a ed. Brasília: Ministério da Saúde; 2008.

12. Ministério da Saúde. Secretaria de Vigilância em Saúde. A malária no Brasil: situação epidemiológica da malária no Brasil 2005. Brasília: Ministério da Saúde; 2005.

13. Ministério da Saúde. Departamento de Informação e Informática do Sistema Único de Saúde. DATASUS. Informações de saúde. [Acesso em 30 maio 2009. Disponível em http://w3.datasus.gov.br/datasus/index.php/.

14. Atanaka-Santos M, Czeresnia D, Souza-Santos R, Oliveira RM. Comportamento epidemiológico as malária no Estado de Mato Grosso, 1980-2003. Rev Soc Bras Med Trop 2006; 39:187-192.

15. Atanaka-Santos M, Souza-Santos R, Czeresnia D. Spatial analysis for stratification of priority malaria control areas, Mato Grosso State, Brazil. Cad Saúde Pública 2007; 23:1099-1112.

16. Chaves SS, Rodrigues LC. An initial examination of the epidemiology of malaria in the state of Roraima, in the Brazilian Amazon Basin. Rev Inst Med Trop Sao Paulo 2000; 42:269-275.

17. Instituto Brasileiro de Geografia e Estatística. Unidades Territoriais de cada unidade da Federação - Estado do Tocantins. [Acesso em 30 maio 2009]. Disponível em http://www.sidra.ibge. gov.br/bda/territorio/default. asp? $\mathrm{z}=\mathrm{t} \& \mathrm{o}=4 \& \mathrm{i}=\mathrm{P} /$.

18. Renault CS, Bastos FA, Filgueira JPPS, Filgueira JPPS, Homma TK. Epidemiologia da malária no Município de Belém - Pará. Rev Paraense Med 2007; 21:19-23.

19. Machado RLD, Couto AARA, Cavasini CE, Calvosa VSP. Malária em região extra-Amazônica: situação no Estado de Santa Catarina. Rev Soc Bras Med Trop $2003 ; 36: 581-586$

20. Coura JR, Suárez-Mutis M, Ladeira-Andrade S. A new challenge for malaria control in Brazil: asymptomatic Plasmodium infection - A Review. Mem Inst Oswaldo Cruz 2006; 101:229-237.

21. Salcedo JMV, Camargo EP, Krieger H, Silva LHP, Camargo LMA. Malaria contro in an agro-industrial settlement of Rondônia (Western Amazon Region, Brazil). Mem Inst Osvaldo Cruz 2000; 95:139-145.

22. Souza-Santos R, Oliveira MVG, Escobar AL, Santos RV, Coimbra-Junior CEA Spatial heterogeneity of malaria in Indian reserves of Southwestern Amazônia, Brazil. Int J Health Geogr 2008; 7:1-10.

23. Oliveira-Filho AB, Martinelli JM. Casos notificados de malária no Estado do Pará Amazônia Brasileira, de 1998 a 2006. Epidemiol Serv Saude 2009; 18:277-284.

24. Fernández LD, Alas JC, Juarez JF, Hernández CA, Santos AG, Cruz OS, et al Índices maláricos como factores de riesgo en el Departyamento del Petén Norte, Guatemala. Rev Cubana Med Trop 2008; 60:148-158.

25. Monte-Mor RLM. Malária e meio ambiente na Amazônia brasileira. In: Seminário Latino-Americano Sobre População e Saúde, 1985, Campinas. População e saúde. Campinas: Unicamp, 1986; 2:312-328.

26. Silveira AC, Rezende DF. Avaliação da estratégia Global de Controle integrado da malária no Brasil. Organização Pan-Americana de Saúde. Brasília; 2001.

27. Yeshiwondim AK, Gopal S, Hailemariam AT, Dengela DO, Patel HP. Spatial analysis of malaria incidence at the village level in areas with unstable transmission in Ethiopia. Int J Health Geogr 2009; 8:1-11.

28. Marques AC, Gutierrez HC. Combate à malária no Brasil: evolução, situação atual e perspectivas. Rev Soc Bras Med Trop 1994; 27:91-108.

29. Forattini OP, Kakitani I, Massad E, Marucci D. Studies on mosquitoes (Díptera: Culicidae) and anthropic environment. 2- Immature reserch at a rice irrigation system location in South-Eastern Brazil. Rev Saude Publica 1993; 27:227-236.

30. Alves MJCP, Mayo RC, Donalisio MR. História, epidemiologia e controle da malária na região de Campinhas, Estado de São Paulo, Brasil, 1980 a 2000 . Rev Soc Bras Med Trop 2004; 37:41-45.

31. Barbieri AF. Uso antrópico da terra e malária no norte de Mato Grosso, 1992 a 1995. 2000. [Dissertação de Mestrado]. [Belo Horizonte (BH)]: Universidade Federal de Minas Gerais; 2000.

32. Marques AC, Pinheiro EA, Souza AG. Um estudo sobre dispersão de casos de malária no Brasil. Rev Bras Malariologia e Doenças Tropicais 1986; 38:51-75.

33. Ministério da Saúde. Secretaria de Vigilância em Saúde. Situação epidemiológica da malária no Brasil 2006. Brasília; 2006.

34. Ministério da Saúde. Secretaria de Vigilância em Saúde. Situação epidemiológica da malária no Brasil 2007. Brasília, 2007. [Acesso em 1º dez. 2007]. Disponível em http://www.saude.gov.br/svs/.

35. Ministério da Saúde. Secretaria de Vigilância em Saúde. Programa Nacional de Controle da Malária - PNCM. 2a ed. rev. Brasília: Ministério da Saúde; 2004 . 